\title{
Kongresse und Fortbildungsveranstaltungen
}

\section{Jahrestagung der Österreichischen Gesellschaft für Innere Medizin} gemeinsam mit der österr. Gesellschaft für Diabetologie österr. Gesellschaft für Kardiologie österr. Gesellschaft für Klinische Chemie österr. Gesellschaft für Laboratoriumsmedizin österr. Gesellschaft für Nuklearmedizin österr. Gesellschaft für Infektionskrankheiten

Salzburg, Kongreßhaus 20.-22. September 1990

7. Jahrestagung der Österreichischen Gesellschaft für Senologie Fortbildungsinstitut der Oberösterr. Landwirt-schaftskammer Auf der Gugl 3, A-4020 Linz 12. und 13. Oktober 1990 First European Conference Biomedical Engineering Nice, 17-20 February 1991 1990 Joint Congress of the International Society of Blood Transfusion (ISBT) and the American Association of Blood Banks (AABB)

November 10-15,1990

Los Angeles Convention Center

Los Angeles, California

For further information, contact AABB Meeting

Services

1117 N. 19th Street, Suite 600, Arlington, VA 22209

Telephone: 703-247-0520.

MEDICA '90, Baden-Baden 23.7.-3.8.1990

MEDICA ‘90, Düsseldorf 21.-24.11.1990

Second International Conference on Shock

5th Meeting of European Shock Society

14 th Annual Meeting of the Shock Society (USA)

3rd Vienna Shock Forum

Tagungsthema:

Polytrauma

Sepsis and Organ failure

Therapeutic Interventions - Sepsis and Organ Failure

Hilton, Wien, 2.-6. Juni 1991 